\title{
Cerebral Candida albicans Infection in Two dogs
}

\author{
Erick Platiní Ferreira de Souto', Maria Talita Soares Frade', Ismael Lira Borges', Leonardo Mendes Torres², \\ Glaucia Denise Kommers ${ }^{3}$, Glauco José Nogueira de Galiza', Franklin Riet-Correa ${ }^{4}$ \& Antônio Flávio Medeiros Dantas'
}

\begin{abstract}
Background: Candida spp. are pleomorphic fungi that are commensal inhabitants of the oral, gastrointestinal, upper respiratory and urogenital mucosa of mammals. Candida albicans is described as the most important species. This opportunistic pathogen may produce local or systemic infections in dogs. Local infections have been reported in several tissues and systemic infection is rare in dogs with few reports in the literature describing this presentation. The aim of the present study was to report two cases of cerebral Candida albicans infection in dogs in Brazil.

Case: Two cases of cerebral Candida albicans infection in dogs that showed nervous signs are described. In both cases, the brain showed marked asymmetry of the telencephalic hemispheres with multifocal to coalescing yellowish or reddish areas and a partial loss of distinction between gray and white matter. In Case 1, the mediastinal, tracheobronchial and mesenteric lymph nodes, as well as the right kidney and adrenal gland, showed altered architecture due to numerous whitish gray nodules. Histological lesions were characterized by granulomatous (case 1) or pyogranulomatous (case 2) necrotizing meningoencephalitis with intralesional fungal organisms. In case 1, similar granulomatous infiltrate with intralesional fungal organisms was also seen in the lymph nodes, kidney and adrenal gland. In case 2, there was evidence consistent with an underlying infection of canine distemper virus. Were observed lymphoplasmocytic interstitial pneumonia, lymphoid rarefaction in lymph nodes, and viral intracytoplasmic inclusion bodies in the epithelial cells of the stomach and vesical urothelium. In these two cases, the fungal organisms displayed three different morphological patterns. The first pattern was characterized by delicate tubular structures with thin parallel walls that were rarely septate and tended to undulate slightly, and measured approximately 4-20 $\mu \mathrm{m}$ (true hyphae). The second pattern was characterized by chains of elongated yeast, separated by constrictions at septal sites, and measured approximately 4-10 $\mu \mathrm{m}$ (pseudohyphae). The other morphological pattern, which was rarely observed, was characterized by round budding yeast cells that measured 3-4 $\mu \mathrm{m}$ in diameter and were often elongated by the germ tube. Sections were also stained using immunohistochemical antibodies against Candida albicans. The fungi revealed strong immunolabelling of the cytoplasm and wall for Candida albicans.

Discussion: The diagnosis was based on the histomorphological and immunohistochemical characteristics of the agent, which were consistent with Candida albicans. In these two cases, immunocompromise appeared to have been an important factor in the progression of the infection. In case 1, the dog was senile and treated with corticosteroids, conditions that could effectively limit innate, humoral and cell-mediated immune response to infection. In case 2, the dog displayed cachexia and was infected by canine distemper virus, evidenced by the presence of viral inclusion bodies. The inflammatory reactions in both cases were characteristic of fungal infections, but were distinct due to differences in the infectious process. In case 1, there was a subacute to chronic progression; while in case 2 neutrophilic infiltrate predominated, suggesting an acute progression. The different morphological patterns of the fungi suggested a pleomorphic fungi and the immunohistochemistry allowed us to identify the infectious agent. In conclusion, opportunistic infections caused by Candida albicans should be considered when diagnosing diseases that affect the central nervous system of dogs, particularly in immunosuppressed animals.
\end{abstract}

Keywords: dog diseases, fungal encephalitis, opportunistic fungus. 


\section{INTRODUCTION}

Candida spp. are pleomorphic fungi that are commensal inhabitants of the oral, gastrointestinal, upper respiratory and urogenital mucosa of mammals. This genus includes nearly 200 species, of which 20 have been implicated as infectious agents in humans and animals [14], with Candida albicans being the most important species [17]. This opportunistic pathogen may produce local or systemic infections in dogs. Local infections have been reported in the skin, external ear, perineum, nail folds, oral mucosa, cornea, urinary tract and gastrointestinal tract [10]. Systemic infection is rare in dogs with few reports in the literature describing this presentation $[1,4,7,9,13,15]$. The aim of the present study was to report two cases of cerebral Candida albicans infection in dogs in Brazil.

\section{CASES}

Two dogs were obtained for clinical evaluation in the Veterinary Hospital of the Federal University of Campina Grande (UFCG). In case 1, a 14-year-old female Poodle domiciled displayed tremors in the hind limbs. A neurological examination was made but was found to be inconclusive. The dog was treated with an analgesic and prednisolone for 5 days. Approximately 12 days later, the dog displayed repetitive movements of the head and rubbed its paws over its ears. It was treated with gentamicin, miconazole, and prednisolone for ten days. At the end of treatment, the clinical condition worsened, and the dog displayed lateral deviation of the head, staggering gait, paddling movements and seizures followed by death. In case 2, an errant male dog mixed breed approximately 5-month-old was found showing clinical signs of apathy, cachexia and incoordination, and died shortly after.

Both dogs were necropsied in the Laboratory of Animal Pathology of the UFCG. In case 1: the brain showed marked asymmetry of the telencephalic hemispheres by diffuse enlargement of the right hemisphere, which displayed a yellowed and thickened gyrus (Figure 1). In the serial sections, the right hemisphere was enlarged, friable, had a rough surface, and lacked distinction between gray and white matter. The lesion extended through the frontal, parietal, temporal and right occipital lobes, affecting the cingulate gyrus, corpus callosum, fornix, basal nuclei, lateral ventricle and hippocampus. The thalamus, third ventricle, mesencephalic aqueduct, and colliculi were compressed.
The mediastinal, tracheobronchial, and mesenteric lymph nodes were enlarged and showed altered architecture due to the presence of numerous whitish gray nodules. The kidneys were diffusely reddish and the right kidney displayed a dark red and firm focal area in the cortico-medullary region. The right adrenal gland was enlarged and showed a small whitish gray nodule in the medullary region. The left and right atrioventricular valves displayed endocardiosis. In case 2: the brain showed moderate asymmetry of the telencephalic hemispheres by enlargement of the right hemisphere. In the serial sections, it was found enlargement of the frontal, parietal and right temporal lobes, which presented multifocal to coalescing reddish areas and partial loss of distinction between gray and white matter (Figure 2). The lungs were diffusely reddish and not collapsed.

Samples from the central nervous system, lymph nodes and organs within the thoracic and abdominal cavities were fixed in $10 \%$ neutral buffered formalin, processed routinely and embedded in paraffin. All sections were stained with haematoxylin and eosin (HE) and selected sections were stained with Grocott's methenamine silver stain (GMS) and periodic acid-Schiff (PAS).

Immunohistochemistry (IHC) was performed to identify the infectious agent in samples from the central nervous system. It were used the monoclonal antibody anti-Aspergillus spp. (WF-AF-1) (Bio-Rad $\left.\operatorname{AbD~Serotec}^{\circledR}\right)^{1}$ at a dilution of 1:300 and the polyclonal antibody anti-Candida albicans (ab53891) (AB$\left.\mathrm{CAM}^{\circledR}\right)^{2}$ at a dilution of 1:1000 and incubated them for $1 \mathrm{~h}$ at $37^{\circ} \mathrm{C}$. An endogenous peroxidase blocking step was performed using $3 \%$ hydrogen peroxide $(2 \mathrm{x}$ $10 \mathrm{~min}$ ). To decrease non-specific binding, the histological sections were treated with $5 \%$ casein solution for $30 \mathrm{~min}$. For antigen retrieval, was used a $\mathrm{pH} 9.0$ tris-EDTA solution. Amplification was performed with Easy Link One polymer (EasyPath $\left.{ }^{\circledR}\right)^{3}$ for $20 \mathrm{~min}$ at $25^{\circ} \mathrm{C}$. The DAB liquid (3,3 'diaminobenzidine) + substrate-Choromogen System $\left(\text { Dako }^{\circledR}\right)^{4}$ was used as the chromogen. As a positive control, it were utilized histological sections of cases previously confirmed for candidiasis. As a negative control, histological sections of each case were incubated with phosphate buffered saline containing $0.5 \%$ Tween 20 (PBST).

Histological lesions in both cases were characterized by granulomatous (case 1) [Figure 3] or 
pyogranulomatous (case 2) severe, multifocal to coalescing necrotizing meningoencephalitis, associated with pale fungal organisms in the HE-stained sections (Figure 3).

In case 1: the lesion extended throughout the entire right hemisphere of the brain including the meninges, gray matter and white matter, and affecting ependymal cells, the lateral ventricle and the choroid plexus. The inflammatory infiltrate consisted of epithelioid macrophages and multinucleated giant cells that were associated with necrosis and intralesional fungal organisms. These areas were occasionally surrounded by mild infiltrate of neutrophils, plasma cells and lymphocytes. There was vasculitis and fibrinoid necrosis in the walls of blood vessels, occasionally associated with fungal organisms. Gitter cells and axonal spheroids were observed near the necrotic areas.

Granulomatous inflammation and necrosis with intralesional fungal organisms were also seen in the mediastinal, tracheobronchial and mesenteric lymph nodes, kidney and adrenal gland, which displayed macroscopic alterations. These organs exhibited diffuse infiltration of macrophages and multinucleated giant cells mixed with occasional neutrophils, lymphocytes, and plasma cells. The multinucleated giant cells often contained fungal elements in their cytoplasm.

In case 2: the lesions were more severe in the frontal and parietal lobes of the brain. The meninges and gray matter were thickened by inflammatory infiltrate comprising epithelioid macrophages, neutrophils and multinucleated giant cells. The white matter showed multifocal to coalescing areas of necrosis, infiltration by neutrophils, intralesional fungal organisms, and hemorrhages. Epithelioid macrophages, multinucleated giant cells, lymphocytes and plasma cells were observed only occasionally in the white matter. It was also observed perivascular inflammatory infiltrate, vasculitis, neuropil vacuolization, myelin degeneration, gitter cells in the periphery of necrotic areas, neuronal degeneration, axonal spheroids, and perivascular and perineuronal edema.

Additionally, case 2 showed lymphoplasmocytic interstitial pneumonia, lymphoid rarefaction in lymph nodes, and viral intracytoplasmic inclusion bodies in the epithelial cells of the stomach and vesical urothelium, which are consistent with a canine distemper virus infection.

In these two cases, the fungal structures displayed three different morphological patterns in the central nervous system sections stained by PAS and GMS. The first pattern was characterized by delicate tubular structures with thin parallel walls that were rarely septate and tended to undulate slightly, and measured approximately 4-20 $\mu \mathrm{m}$ (true hyphae) [Figure 4A]. The second pattern was characterized by chains of elongated yeast, separated by constrictions at septal sites, and measured approximately 4-10 $\mu \mathrm{m}$ (pseudohyphae) [Figure 4B]. The other morphological pattern, which was rarely observed, was characterized by round budding yeast cells that measured 3-4 $\mu \mathrm{m}$ in diameter and were often elongated by the germ tube (Figure 4C). True hyphae and pseudohyphae were predominant. Sections were also stained using immunohistochemical antibodies against Aspergillus spp. and Candida albicans. The fungi revealed negative immunolabelling for Aspergillus spp. and strong immunolabelling of the cytoplasm and wall for Candida albicans (Figure 4D).

\section{DISCUSSION}

The diagnosis of candidiasis was based on the fungi's histomorphological and immunohistochemical characteristics, which were consistent with Candida albicans. This opportunistic pathogen usually requires impairment of the host defense mechanisms to establish clinical infection [10].

In these two cases, immunocompromise appeared to have been an important factor in the progression of the infection. In case 1, the dog was senile and treated with corticosteroids, conditions that could effectively limit innate, humoral and cell-mediated immune response to infection [16]. In case 2, the dog displayed cachexia and was infected by canine distemper virus, evidenced by the presence of viral inclusion bodies. This virus induces lymphocytic apoptosis, lymphoid depletion and consequently immunosuppression, predisposing the host to secondary infections [2].

Considering the preexisting colonization of the oral, gastrointestinal, upper respiratory and urogenital mucosa by commensal Candida spp. [14], the portal of entry is difficult to establish in both cases. It is likely that tissue invasion by Candida occurs through the gastrointestinal mucosa, epithelially denuded surfaces, contaminated intravascular devices, trauma, or surgically related inoculation [6].

Both dogs showed clinical signs of neurological alterations. In case 1, a neurological examination was 


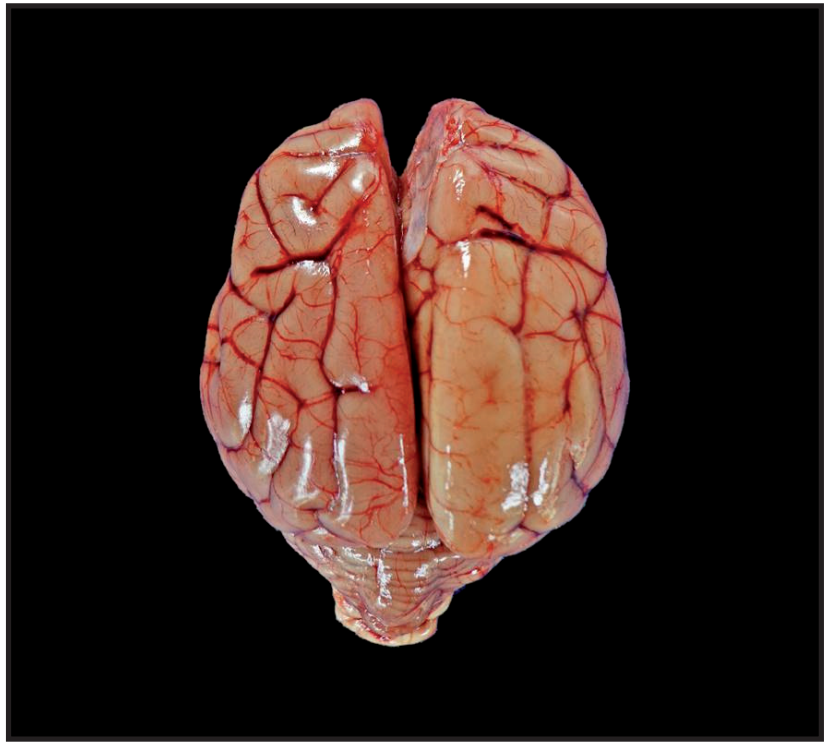

Figure 1. Cerebral Candida albicans infection in dog. Case 1. Asymmetry of the telencephalic hemispheres by diffuse enlargement of the right hemisphere, which displayed a yellowed and thickened gyrus.

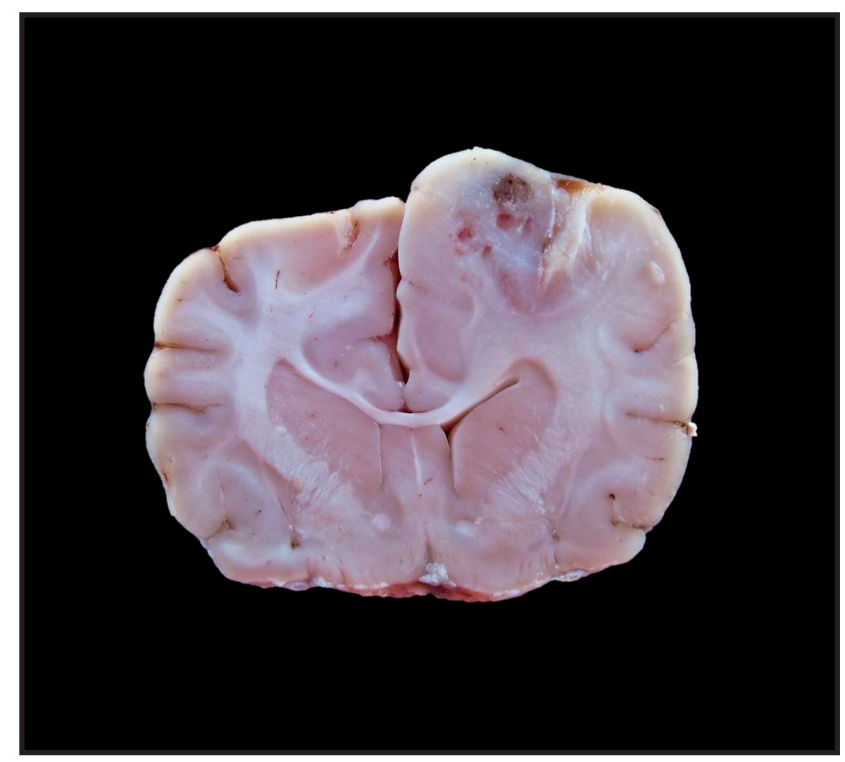

Figure 2. Cerebral Candida albicans infection in dog. Case 2. Cross section of the brain, after fixation, showing enlargement of the parietal and temporal right lobe, which presented multifocal to coalescing reddish areas and partial loss of distinction between gray and white matter.

performed, but no significant neurological changes were observed, probably due to the initial phase of the infection. Because the treatment was ineffective, it was possible to observe the progression of neurological signs characterizing the diffuse damage to the right cerebral hemisphere. In case 2, we were unable to obtain detailed information about the clinical evolution because it was an errant animal that died before an appropriate clinical evaluation. Nevertheless, while case 2 was characterized by an infection restricted to the brain, the infection in case 1 spread to the lymph nodes and abdominal cavity organs, which is indicative of a systemic infection.

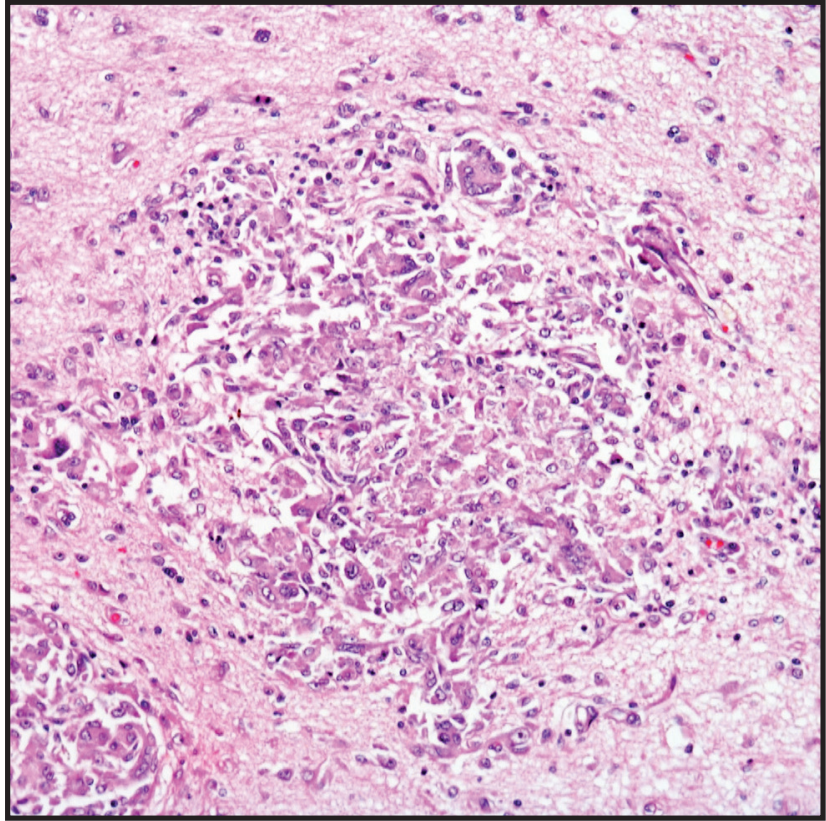

Figure 3. Cerebral Candida albicans infection in dog. Case 1. Granulomatous meningoencephalitis with multinucleated giant cells [HE; Bar=50 $\mu \mathrm{m}$ ].

Most reports of systemic candidiasis in dogs have been associated with immunosuppressive conditions, such as long-term corticosteroid therapy, cytotoxic chemotherapy, malnutrition, diabetes mellitus [7] and previous parvovirus infection [13]. Systemic candidiasis was also observed in dogs with neutropenia induced by cyclophosphamide [8]. However, there are some reports in which the affected dogs did not present an underlying associated condition $[1,9,13,15]$.

The inflammatory reactions in both cases were characteristic of fungal infections, but were distinct due to differences in the infectious process. In case 1, there was a predominance of mononuclear inflammatory infiltrate, suggesting a subacute to chronic progression; while in case 2 neutrophilic infiltrate predominated, suggesting an acute progression.

The different morphological patterns of the fungi, best visualized with GMS and PAS stains, suggested a pleomorphic fungi. A few species of Candida actively respond to the host's immune mechanisms by changing morphotypes, from yeast form, typical for commensal Candida, to a more resistant form with germ tubes, pseudohyphae and/or true hyphae, typical of established candidiasis [11]. The switch from the yeast to hyphal morphotypes is an important factor of virulence and survival [11].

Due the pleomorphism in the fungal structures, we do not consider yeast as a significantly differential diagnosis. The predominance of the hyphal 


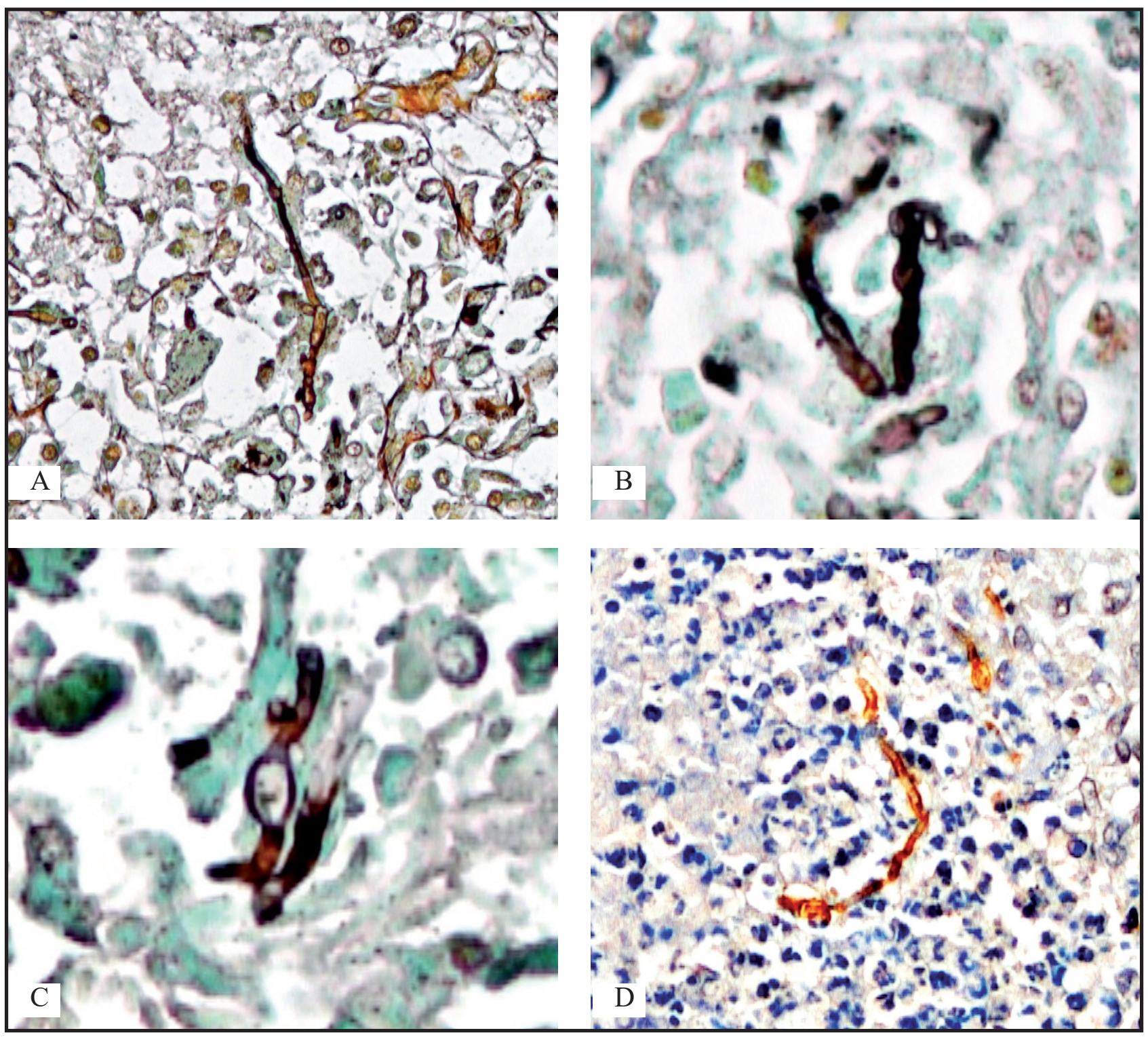

Figure 4. Cerebral Candida albicans infection in dog. Sections of the central nervous system. A- True hyphae [GMS; Bar= $20 \mu \mathrm{m}]$. B- Two pseudohyphaes [GMS; Bar $=20 \mu \mathrm{m}$ ]. C- Yeast cell elongated by the germ tube [GMS; Bar= $20 \mu \mathrm{m}$ ]. D- Strong immunolabelling of true hyphae, in brown [ IHC, method HRP-polymer; Bar= $20 \mu \mathrm{m}]$.

morphotypes suggests as differential diagnosis fungi of the genus Aspergillus and Penicillium, which can be differentiated from the fungi of the genus Candida by their particular histomorphological characteristics. Another important differential diagnosis is with infection by bacteria of the genus Nocardia, which are long filamentous organisms with a tendency to fragment into cocobacillary forms, best viewed when stained with Gram, Giemsa and acid-fast (modified Ziehl-Neelsen) stains [12].

In conclusion, opportunistic infections caused by Candida albicans should be considered when diagnosing diseases that affect the central nervous system of dogs, particularly in immunosuppressed animals.

\section{MANUFACTURERS}

\author{
${ }^{1}$ Bio-Rad AbD Serotec. São Paulo, SP, Brazil. \\ ${ }^{2}$ abcam. Cambridge, MA, USA. \\ ${ }^{3}$ EasyPath. São Paulo, SP, Brazil. \\ ${ }^{4}$ Dako. Glostrup, Denmark.
}

Acknowledgments. The authors are grateful to the National Research Council (CNPq) and to the productivity scholarship, process number 302082/2014-0.

Declaration of interest. The authors report no conflicts of interest. The authors alone are responsible for the content and writing of the paper. 


\section{REFERENCES}

1 Brown M.R., Thompson C.A. \& Mohamed F.M. 2005. Systemic candidiasis in an apparently immunocompetent dog. Journal of Veterinary Diagnostic Investigation. 17(1): 272-276.

2 Caswell J.L. \& Williams K.J. 2016. Respiratory system. In: Maxie M.G. (Ed). Jubb, Kennedy, and Palmer's Pathology of Domestic Animals. v2. 6th edn. Saint Louis: Elsevier, pp.465-591.

3 Chen C., Su C.H. \& Lee L.W. 2008. Systemic candidiasis due to Candida albicans in a dog. The Japanese Journal of Veterinary Dermatology. 13(21): 211-229.

4 Clercx C., Meentee K., Snaps F., Jacquinet E. \& Coignoul F. 1996. Bronchopulmonary and disseminated granulomatous disease associated with Aspergillus fumigatus and Candida species infection in a golden retriever. Journal of the American Animal Hospital Association. 32(12): 139-145.

5 Day M.J., Peeters D. \& Clercx C. 2012. Aspergillosis and Penicilliosis. In: Greene C.E. (Ed). Infectious Diseases of the Dog and Cat. 4th edn. Saint Louis: Elsevier, pp.1354-1370.

6 Grubb S.E., Murdoch C., Sudbery P.E., Saville S.P., Lopez-Ribot J.L. \& Thornhill M.H. 2008. Candida albicans endothelial cell interactions: a key step in the pathogenesis of systemic candidiasis. Infection and Immunity. 76(11): 4370-4377.

7 Heseltine J.C., Panciera D.L. \& Saunders G.K. 2003. Systemic candidiasis in a dog. Journal of the American Veterinary Medical Association. 223(5): 821-824.

8 Khosravi A.R., Mardjanmehr H., Shokri H., Naghshineh R., Rostamibashman M. \& Naseri A. 2009. Mycological and histopathological findings of experimental disseminated candidiasis in dogs. Iranian Journal of Veterinary Research. 10(4): 228-234.

9 Kuwamura M., Ide M., Yamate J., Shiraishi Y. \& Kotani T. 2006. Systemic candidiasis in a dog, developing spondylitis. Journal of Veterinary Medical Science. 68(6): 1117-1119.

10 Pressler B.M. 2012. Candidiasis and rhodotorulosis. In: Greene C.E. (Ed). Infectious Diseases of the Dog and Cat. 4th edn. Saint Louis: Elsevier, pp.666-672.

11 Raska M., Belakova J., Krupka M. \& Weigl E. 2007. Candidiasis. Do we need to fight or to tolerate the Candida fungus. Folia Microbiologica. 52(11): 297-312.

12 Ribeiro M.G., Salerno T., Mattos-Guaraldi A.L., Camello T.C.F., Langoni H., Siqueira A.K., Paes A.C., Fernandes M.C. \& Lara G.H.B. 2008. Nocardiosis: an overview and additional report of 28 cases in cattle and dogs. Revista do Instituto de Medicina Tropical de São Paulo. 50(2): 177-185.

13 Rodríguez F., Fernández A., Espinosa de los Monteros A., Wohlsein P. \& Jensen H.E. 1998. Acute disseminated candidiasis in a puppy associated with parvoviral infection. Veterinary Record. 142(2): 434-436.

14 Segal E. 2005. Candida, still number one. What do we know and where are we going from there. Mycoses. 48(48): 03-11.

15 Skoric M., Fictum P., Slana I., Kriz P. \& Pavlik I. 2011. A case of systemic mycosis in a Hovawart dog due to Candida albicans. Veterinaria Medicina. 56(8): 260-264.

16 Tizard I.R. 2008. Imunodeficiências secundárias. In: Tizard I.R. (Ed). Imunologia Veterinária. 8.ed. Rio de Janeiro: Elsevier, pp.473-488.

17 Uzal F.A., Plattner B.L. \& Hostetter J.M. 2016. Alimentary system. In: Maxie M.G. (Ed). Jubb, Kennedy, and Palmer's Pathology of Domestic Animals. 6th edn. Saint Louis: Elsevier, pp.1-257. 